IRSTI 27.35.17

\author{
${ }^{1}$ A. Beketayeva, ${ }^{2}$ A.Zadauly \\ ${ }^{1}$ Institute of Mathematics and Mathematical Modeling \\ of Ministry of Education and Science of Republic of Kazakhstan, Almaty, Kazakhstan \\ e-mail: azimaras10@gmail.com \\ ${ }^{2}$ Institute of Mechanics and Engineering Science \\ of Ministry of Education and Science of Republic of Kazakhstan, Almaty, Kazakhstan \\ e-mail: akerkeh_333@mail.ru
}

\title{
Influence of slit sizes on the interaction structure of supersonic turbulent air flow with a multi-component injection jet in a channel
}

\begin{abstract}
The supersonic air flow in a flat channel with transverse injection of a turbulent hydrogen jet through a slit in the bottom wall is numerically simulated. The solution of the initial Favre-averaged Navier-Stokes equations closed by the k- $\omega$ turbulence model is performed using an algorithm built on the basis of the WENO scheme. The interaction of a shock-wave structure with boundary layers on the lower and upper walls under conditions of an internal turbulent flow is investigated, namely, the effect of the width of the jet slit is studied. It is found that, in addition to the known shock-wave structures arising from the interaction of the incident flow with the transverse jet and the interaction of the main shock wave with the boundary layers near the walls, there is an additional system of shock waves and separation of the flow on the bottom wall at some distance from the jet downwards flow. Comparison with experimental data showed satisfactory agreement.
\end{abstract}

Key words: supersonic flow, perfect gas, boundary layer, the Navier-Stokes equations.

\section{Introduction}

Most published theoretical studies of the interaction of the jet and the oncoming flow in the channel mainly consider the jet injection region and generally the boundary conditions of symmetry or free flow are realized at the upper boundary [1-3], which greatly simplifies the solution of an assigned task. For example, in [1] the processes of mixing and chemical reactions during the transverse injection of a sound jet of hydrogen into a supersonic air stream with the parameters of $M_{\infty}=4, T=1000 \mathrm{~K} p=4 \mathrm{~atm}$ were calculated. Some features of the emerging flow were identified, including establishing the existence of the reverse current zones in front of the injector and behind it and obtaining the distributions of concentrations of the mixture of components. Studies in [2] dealt with the transverse injection of gaseous fuel at an angle into a low elongated supersonic combustion channel. It is shown numerically that the fuel injection at an angle leads to an increase in the efficiency of mixing of fuel with air.The authors of [3] considered the flow of a multicomponent gas mixture with the injection of a helium jet through a circular opening at different angles of inclination $\left(30^{\circ}, 90^{\circ}\right.$ and $\left.150^{\circ}\right)$. The influence of the angle of inclination of the injecting jet on a supersonic gas mixing was studied. It was found that the larger the angle, the greater the overall coefficient of pressure loss in the jet.

The mechanism of formation of the shockwave structure of the aforementioned flow is well described in the literature [1-7]. A schematic picture of the flow in the injection region is shown in Figure 1 , where 1 is the head shock wave resulting from the 
deceleration of the oncoming flow in front of the jet, 2 and 3 are oblique and closing shock waves, respectively.The intersection of the head, oblique and closing shock waves at one point is the $\lambda$-shaped structure. The diagram depicts vorticesin front of the jet formed as a result of the primary and secondary separation of the flow from the wall. In this case, the vortex, located closer to the jet, moves counterclockwise and the vortex located at a greater distance from it, moves clockwise [4-6]. The figure also shows the detachable area behind the jet which occurs due to the discharge zone. In addition, the flow diagram (Figure 1) shows a weak shock, which is formed behind the injection nozzle when a stream of airflow that has passed around the blown jet flows onto the wall.

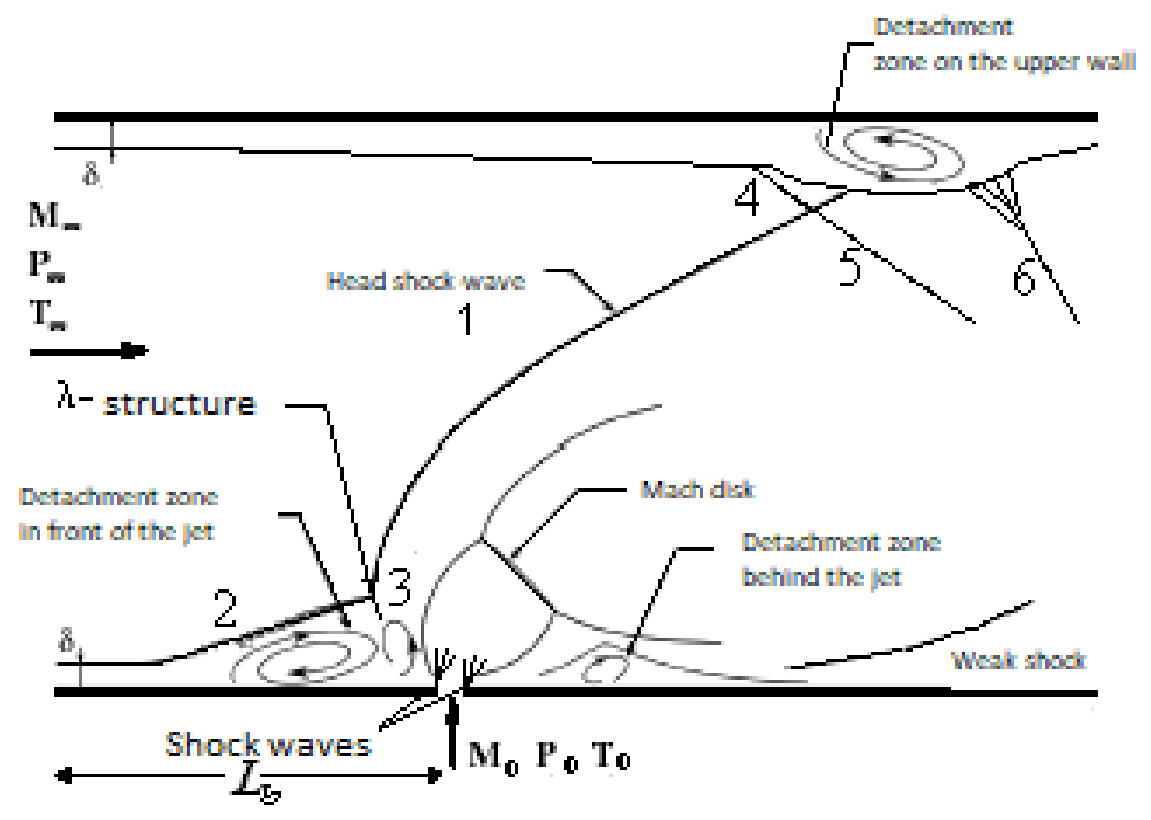

Figure 1 - The flow diagram

In some papers, the interaction of a transverse jet blown from the bottom wall and a shock wave falling from the upper wall of the channel was considered which also somewhat simplifies the formulation of the problem and allows a detailed study of their interaction. Thus in [8], the interaction of afalling shock wave and a transverse jet in a supersonic flow was numerically studied. The influence of the size and location of the shock wave generator falling from the top wall on the pressure profiles on the bottom wall in the region of the jet injection was studied. The inclination angle of the generator ranged from $15,784^{\circ}$ to $35,974^{0}$. The authors showed that not only the inclination angle, but also the size of the generator significantly increases the interaction area both in front of the jet and behind it.In [9] the results of a study of the influence of shock waves on supersonic burning of a hydrogen jet were given, and the main attention was on the growth of the mixing layer. The effect of the falling shock wave on the turbulent boundary layer was numerically investigated in [10] where the intensity of the shock wave was changed by adjusting the angle of the shock wave generator. Such models of turbulence as the k- $\varepsilon, \mathrm{k}-\varepsilon / \mathrm{k}-\omega$ hybrid model and the model with one equation for turbulent viscosity were studied in the work.

Experimental and theoretical papers studied the interaction of the falling shock wave but with the boundary layer [11-12]. There are almost no studies devoted to the interaction of a shock wave with a boundary layer under conditions of an internal turbulent flow. A schematic picture of the interaction of the shock wave with the boundary layer on the upper wall is also shown in Figure 1. Here 1 is the head shock wave falling on the upper wall, 4 is the shock wave separating the detachment region on the upper wall from the supersonic oncoming stream, 5 is the reflected shock wave and 6 is the compression wave arising at the point of 
attachment of the detached flow. Thus, in [11] the results of calculations obtained by the method of large eddies are presented. The focus is on studying the structure of the flow, the size of the detachment zone, as well as the dynamic and thermal loads that occur during this interaction. The conditions under which the angle of inclination and the intensity of the shock wave lead to the separation of the boundary layer were investigated in [12].

The practical interest of supersonic flow with injection of jets in a channel is associated with the arising loads on both the upper and lower walls of the channel. The structure of the interaction will depend on the parameters of the injection jet, as well as on the height of the channel itself. As mentioned above, the problems of interaction of a shock wave with the injection jet and with boundary layers are mainly considered separately, not only because of the complexity of the numerical implementation of the nature of the flow and of the gas under consideration, but also with the presence of the upper wall, which requires reliable numerical calculation methods.

The difficulties encountered in the numerical solution of the problems described above do not allow a full study of the structure of both the interaction of the jet with the transverse flow and the interaction of the shock wave with the boundary layers on the lower and upper walls. The main problem of numerical simulation is well reflected in the literature [13-16]. At present, quasi-monotone conservative schemes of a higher approximation order, such as TVD (Total Variation Diminishing Schemes), ENO (Essentially Nonoscillatory Schemes), WENO (Weighted ENO) schemes [1819], are widely used to solve such complex problems. In [16] the authors developed the ENO scheme based on the Godunov method and showed the applicability of the scheme in solving the problem of supersonic flow of a multicomponent gas in a flat channel with injection of perpendicular jets.

The purpose of this paper is the numerical simulation of supersonic flow in a flat channel with transverse injection of a multicomponent jet and the study of the interaction of a shock wave arising in front of the injection jet with a boundary layer on both the lower and upper walls. Also included is the numerical study of the conditions of detachment of the boundary layer on the walls, as well as the influence of the jet width on the occurrence of detachment.

\section{Problem definition}

A supersonic flow of air with a transverse jet injection from the bottom wall of a rectangular channel is shown in Figure 1. The system of twodimensional Favre-averaged Navier-Stokes equations for a multicomponent gas mixture with respect to Cartesian coordinates in a conservative form is represented in the form:

$$
\begin{gathered}
\frac{\partial \vec{U}}{\partial t}+\frac{\partial\left(\vec{E} \vec{E}_{v}\right)}{\partial x}+\frac{\partial\left(\vec{F} \vec{F}_{v}\right)}{\partial z}=0 \\
\vec{U}=\left(\rho, \rho u, \rho w, E_{t}, \rho Y_{k}\right)^{T}, \\
\vec{E}=\left(\rho u, \rho u^{2}+P, \rho u w,\left(E_{t}+P\right) u, \rho u Y_{k}\right)^{T}, \vec{F}=\left(\rho w, \rho u w, \rho w^{2}+P,\left(E_{t}+P\right) w, \rho w Y_{k}\right)^{T}, \\
\vec{E}_{v}=\left(0, \tau_{x x}, \tau_{x z}, u \tau_{x x}+w \tau_{x z}-q_{x}, J_{k x}\right)^{T}, \vec{F}_{v}=\left(0, \tau_{x z}, \tau_{z z}, u \tau_{x z}+w \tau_{z z}-q_{z}, J_{k z}\right)^{T}, \\
P=\frac{\rho T}{\gamma \infty M{ }_{\infty}^{2} W}, \quad W=\left(\sum_{k=1}^{N_{p} Y_{k}}\right)^{-1}, \sum_{k=1}^{N_{p}} Y_{k}=1 \\
E_{t}=\frac{\rho}{\gamma_{\infty} M_{\infty}^{2}} h-P+\frac{1}{2} \rho\left(u^{2}+w^{2}\right), h=\sum_{k=1}^{N_{p}} Y_{k} h_{k}, h_{k}=h_{k}^{0}+\int_{T_{0}}^{T} c_{p k} d T, c_{p k}=C_{p k} / W_{k}
\end{gathered}
$$




$$
\begin{gathered}
\tau_{x x}=\frac{\mu}{\operatorname{Re}}\left(2 u_{x}-\frac{2}{3}\left(u_{x}+w_{z}\right)\right), \tau_{x x}=\frac{\mu}{\operatorname{Re}}\left(2 w_{z}-\frac{2}{3}\left(u_{x}+w_{z}\right)\right), \tau_{x z}=\tau_{z x}=\frac{\mu}{\operatorname{Re}}\left(u_{z}+w_{x}\right), \\
q_{x}=\left(\frac{\mu}{\operatorname{Pr} \operatorname{Re}}\right) \frac{\partial T}{\partial x}+\frac{1}{\gamma_{\infty} M_{\infty}^{2}} \sum_{k=1}^{N_{p}} h_{k} J_{x k}, q_{z}=\left(\frac{\mu}{\operatorname{PrRe}}\right) \frac{\partial T}{\partial z}+\frac{1}{\gamma_{\infty} M_{\infty}^{2}} \sum_{k=1}^{N_{p}} h_{k} J_{z k} . \\
J_{k x}=\frac{\mu}{\operatorname{ScRe} \frac{\partial Y_{k}}{\partial x}}, J_{k z}=-\frac{\mu \partial Y_{k}}{\operatorname{ScRe} \partial z}
\end{gathered}
$$

The equations (1.1) are written in a dimensionless form in the generally accepted notation; the flow parameters $\left(u_{\infty}, \rho_{\infty}, \mathrm{T}_{\infty}\right)$ are accepted as determining ones; the pressure $(\mathrm{P})$ and total energy $\left(E_{t}\right)$ are related to the value $u_{\infty}^{2} \rho_{\infty}$; specific enthalpy $\left(h_{k}\right)$ is related to the value $R T_{\infty} / W_{\infty}$; the molar specific heats $\left(C_{p k}\right)$ are related to $R$; the characteristic length parameter is the slit width. $Y_{k}$ is the mass concentration of $k$ component; the mass concentration index $k=1$ is related to $\mathrm{O}_{2} ; k=2-H_{2}$ (or $k=2-H e), k=3-N_{2}$, and $N_{p}=3$ are the numbers of the gas mixture. $W_{k}$ is the molecular weight of $k$ component; $R e, P r, S c$ are the Reynolds, Prandtl and Shmidt numbers, respectively; $\tau_{x x}, \tau_{z z}, \tau_{x z}, \tau_{z x}$ are viscous stress tensors; $q_{x}, d_{z}, J_{x k}, J_{z k}$ are thermal and diffusion flows (diffusion flows are calculated according to Fick's law); and $\mu=\mu_{l}+\mu_{t}$ are coefficients of laminar and turbulent viscosity. To determine $\mu_{t}$, the $k-\omega$ turbulence model is used.

\section{Boundary conditions}

At the input:

$$
\begin{gathered}
W_{k}=W_{k \infty}, P=P_{\infty}, T=T_{\infty}, u=M_{\infty} \sqrt{\frac{\gamma_{\infty} R_{0} T_{\infty}}{W_{\infty}}}, \\
w=0, Y_{k}=Y_{k \infty}, \quad x=0, \quad 0 \leq z \leq H ;
\end{gathered}
$$

in the input section near the channel walls, the boundary layer is specified, and the longitudinal velocity is approximated by a power law;

at the slit:

$$
W_{k}=W_{k 0}, P=n P_{\infty}, T=T_{0}, w=M_{0} \sqrt{\frac{\gamma_{0} R_{0} T_{0}}{W_{0}}},
$$

$$
u=0, Y_{k}=Y_{k 0}, \quad z=0, \quad L_{b} \leq x \leq L_{b}+h
$$

( $n=P_{0} / P_{\infty}$ is the pressure ratio degree; $P_{0}, P_{\infty}$ are the pressures in the jet and in the flow, respectively; $M_{0}, M_{\infty}$ are the Mach numbers in the jet and in the flow, respectively; indexes of 0 and $\propto$ are related to the parameters of the jet and the flow);

on the lower and upper walls the condition of adhesion and insulation is settled; on the output boundary, nonreflection conditions are specified [17].

\section{Method of solution}

On the lower and upper walls in the boundary layer, as well as at the level of the slit, thickening of the grid is introduced for a more accurate numerical solution. Then the equations (1.1) in the transformed coordinate system are written in the form:

$$
\begin{gathered}
\frac{\partial \widetilde{U}}{\partial t}+\frac{\partial \widetilde{E}}{\partial \xi}+\frac{\partial \widetilde{F}}{\partial \eta}=\frac{\partial \widetilde{E}_{v 2}}{\partial \xi}+\frac{\partial \widetilde{E}_{v m}}{\partial \xi}+\frac{\partial \widetilde{F}_{v 2}}{\partial \eta}+\frac{\partial \widetilde{F}_{v m}}{\partial \eta} \\
\widetilde{U}=\vec{U} / J, \widetilde{E}=\xi_{x} \vec{E} / J, \widetilde{F}=\eta_{z} \vec{F} / J \\
\widetilde{E}_{v 2}=\xi_{x} \vec{E}_{v 2} / J, \widetilde{E}_{v m}=\xi_{x} \vec{E}_{v m} / J \\
\widetilde{F}_{v 2}=\eta_{z} \vec{F}_{v 2} / J, \widetilde{F}_{v m}=\eta_{z} \vec{F}_{v m} / J \\
J=\partial(\xi, \eta) / \partial(x, z)_{\text {-the Jacobian of }} \\
\text { transformation. }
\end{gathered}
$$


The coordinate transformation parameters are described in detail in $[16,18]$.

In this paper convective terms are approximated using the WENO scheme, which is based on the ENO scheme and described in detail in [18]. In the proposed WENO scheme when interpolating a piecewise constant polynomial function, the Newton third-order polynomials are used. Instead of choosing one interpolation polynomial a convex combination is used with the weighting coefficients of all represented polynomials. This achieves a substantially non-oscillating property of the scheme which increases the order of approximation of the scheme. In accordance with [18], a one-step finitedifference scheme for integrating the system (3.1) over time is represented by:

$$
\begin{gathered}
\Delta \widetilde{U}^{n+1}+\Delta t\left[\left(\hat{A}^{+}+\hat{A}^{-}\right) \frac{\partial \vec{E}^{m}}{\partial \xi}+\left(\hat{B}^{+}+\hat{B}^{-}\right) \frac{\partial \vec{F}^{m}}{\partial \eta}-\right. \\
\left.-\left[\frac{\partial\left(\widetilde{E}_{v 2}^{n+1}+\widetilde{E}_{v m}^{n}\right)}{\partial \xi}-\frac{\partial\left(\widetilde{F}_{v 2}^{n+1}+\widetilde{F}_{v m}^{n}\right)}{\partial \eta}\right]\right]=O\left(1 / 2^{\Delta} t^{2}\right)
\end{gathered}
$$

$\hat{A}_{\xi}^{ \pm}, \hat{B}_{\xi}^{ \pm}$are normalized Jacobi matrixes of the next form:

$$
\hat{A}_{\xi}^{ \pm}=R\left(\frac{1 \pm \operatorname{sign}\left(\Lambda_{\xi}\right)}{2}\right) R^{-1}, \hat{B}_{\xi}^{ \pm}=T\left(\frac{1 \pm \operatorname{sign}\left(\Lambda_{\eta}\right)}{2}\right) T^{-1}
$$

$\vec{E}^{m}, \vec{F}^{m}$ are modified flows on the nodal points $(i, j)$ consisting of source convective vectors $(\tilde{E}, \tilde{F})$ and addictive high-order accuracy parameters.
In contrast to the high-order accuracy parameters of [18], vectors $\vec{E}_{\xi i j}, \vec{D}_{\xi i j}$ for positive and negative eigenvalues $\lambda_{i j}$ are written in the next form:

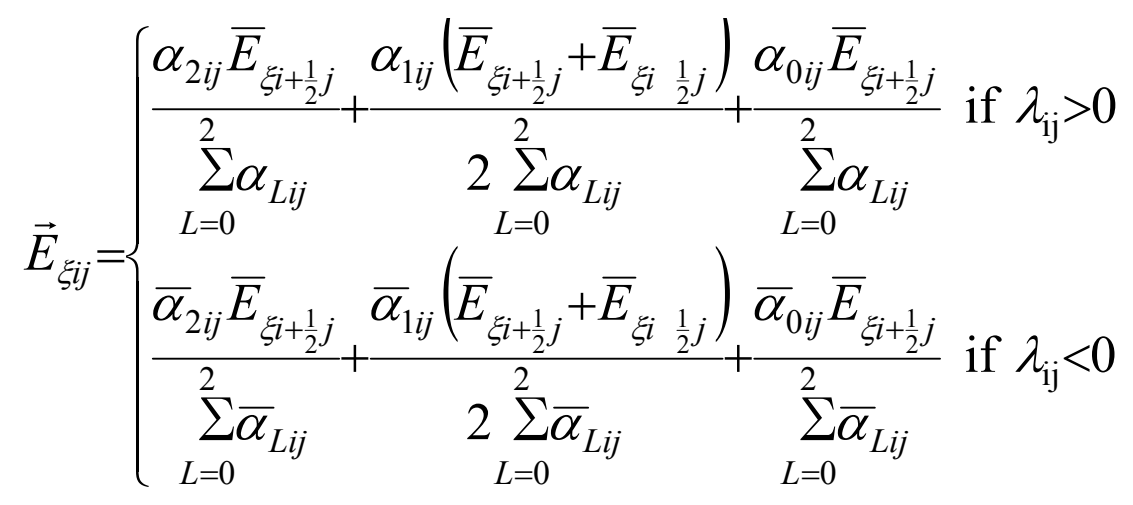

$$
\begin{aligned}
& \vec{D}_{\xi i j}=\left\{\begin{array}{l}
\frac{\alpha_{2 i j} \Delta_{+} \bar{D}_{\xi i+\frac{1}{2} j}}{\sum_{L=0}^{2} \alpha_{L i j}}+\frac{\alpha_{1 i j}\left(\Delta \bar{D}_{\xi i+\frac{1}{2} j}+\Delta_{+} \hat{D}_{\xi i} \frac{1}{2} j\right.}{2}+\frac{\alpha_{0 i j} \Delta \hat{D}_{\xi i} \frac{1}{2} j}{2} \text { if } \lambda_{\mathrm{ij}}>0 \\
\frac{\sum_{L=0}^{2} \alpha_{L i j}}{\alpha_{2 i j} \Delta_{+} \hat{D}_{\xi i+\frac{1}{2} j}}+\frac{\alpha_{1 i j}\left(\Delta \hat{D}_{\xi i+\frac{1}{2} j}^{2}+\Delta_{+} \bar{D}_{\xi i \frac{1}{2} j}\right)}{2 \sum_{L=0}^{2} \alpha_{L i j}}+\frac{\alpha_{0 i j} \Delta \bar{D}_{\xi i \frac{1}{2} j}}{\sum_{L=0}^{2} \alpha_{L i j}} \text { if } \lambda_{\mathrm{ij}}<0
\end{array}\right.
\end{aligned}
$$


where

$$
\begin{gathered}
\bar{E}_{\xi i \pm \frac{1}{2} j}=\left(R \operatorname{sign}\left(\Lambda_{\xi}\right) R^{-1}\right)_{i \pm \frac{1}{2} j}\left[\frac{I-(\Delta t / \Delta \xi)\left(R\left|\Lambda_{\xi}\right| R^{-1}\right)_{i \pm \frac{1}{2} j}}{2}\right] \Delta_{ \pm} \widetilde{E}_{i j}, \\
\bar{D}_{\xi i \pm \frac{1}{2} j}=\left(R \operatorname{sign}\left(\Lambda_{\xi}\right) R^{-1}\right)_{i \pm \frac{1}{2} j}\left[\frac{\left.\left.(\Delta t / \Delta \xi)\left(R\left|\Lambda_{\xi}\right| R^{-1}\right)_{i \pm \frac{1}{2} j}\right]^{2}-I\right]}{2}\right] \Delta_{ \pm} \widetilde{E}_{i j}, \hat{D}_{\xi i \pm \frac{1}{2} j}=\bar{E}_{\xi i \pm \frac{1}{2} j}+\bar{D}_{\xi i \pm \frac{1}{2} j}
\end{gathered}
$$

Values for weight coefficients $\alpha_{L i j}, \bar{\alpha}_{L i j}>0(\mathrm{~L}=0,1,2)$ are obtained in the next form:

$$
\alpha_{L i j}=\frac{C_{L i j}}{\left(\varepsilon+I S_{i+L j}\right)^{3}} \text { и } \bar{\alpha}_{L i j}=\frac{\bar{C}_{L i j}}{\left(\varepsilon+I S_{i+L j}\right)^{3}}
$$

where

$$
C_{0 i j}=\frac{1}{12}, C_{1 i j}=\frac{1}{2}, C_{2 i j}=\frac{1}{4}, \bar{C}_{0 i j}=\frac{1}{4}, \bar{C}_{1 i j}=\frac{1}{2}, \bar{C}_{2 i j}=\frac{1}{12}[19]
$$

and $I S_{i j}$ is an indicator of smoothness of the solution values of the derivatives of the desired and is found by summing all the root-mean-square vector $\tilde{U}$ :

$$
I S_{i j}=\frac{1}{2}\left[\left(\Delta \widetilde{U}_{i-2 j}\right)^{2}+\left(\Delta \widetilde{U}_{i-1 j}\right)^{2}\right]+\left[\Delta^{2} \widetilde{U}_{i-2 j}\right]^{2}
$$

where $\quad \Delta \widetilde{U}_{i j}=\widetilde{U}_{i+1 j}-\widetilde{U}_{i j} \quad$ and $\Delta^{2} \widetilde{U}_{i j}=\Delta \widetilde{U}_{i+1 j}-\Delta \widetilde{U}_{i j}$.

To avoid uncertainty in the denominator of the weight coefficients $\alpha_{L i j}$ and $\bar{\alpha}_{L i j}$ the low coefficient $10^{-7}<\varepsilon<10^{-5}$ is added in (3.6). Vectors $\vec{E}_{\eta i j}$ and $\vec{D}_{\eta i j}$ are written in the same way. Further in the system of equations (3.2), terms containing second derivatives are represented as a sum of two vectors: vectors of second derivatives and vectors of dissipative members and flow vectors with mixed derivatives are approximated explicitly with a second order of accuracy [18]. Linearization of convective terms is carried out using the properties of homogeneity.

After applying factorization to the system (3.2), we have two one-dimensional operators for an implicit solution with respect to the vector of thermodynamic parameters by matrix driving:

1 -st step.

$$
\left[I+\Delta t\left[\left(\widehat{A}_{i-\frac{1}{2} j}^{+} \Delta_{-} A_{\xi i j}^{n}+\widehat{A}_{i+\frac{1}{2} j}^{-} \Delta_{+} A_{\xi i j}^{n}\right)+\Delta\left(\frac{\mu_{t} \xi_{x}^{2}}{\operatorname{Re} J}\right)_{i j} \Delta\left(\frac{1}{U_{1}^{n}}\right)_{i j}\right]\right] U_{i j}^{*}=R H S_{\xi i j}^{n}+R H S_{\eta i j}^{n}
$$


2-nd step.

$$
\left[+\Delta t\left[\left(\widehat{B}_{i j-\frac{1}{2}}^{+} \Delta_{-} B_{\eta i j}^{n}+\widehat{B}_{i j+\frac{1}{2}}^{-} \Delta_{+} B_{\eta j}^{n}\right)+\Delta\left(\frac{\mu_{t} \eta_{z}^{2}}{\operatorname{Re} J}\right)_{i j} \Delta\left(\frac{1}{U_{1}^{n}}\right)_{i j}\right]\right] \widetilde{U}_{i j}^{n+1}=U_{i j}^{*}
$$

were

$$
R H S_{\xi i j}^{n}=\widehat{A}_{i+\frac{1}{2} j}^{-}\left[\left(\vec{E}_{\xi i+1 j}+\vec{D}_{\xi i+1 j}\right)-\left(\vec{E}_{\xi i j}+\vec{D}_{\xi i j}\right)\right]^{n}+\widehat{A}_{i-\frac{1}{2} j}^{+}\left[\left(\vec{E}_{\xi i j}+\vec{D}_{\xi i j}\right)-\left(\vec{E}_{\xi i-1 j}+\vec{D}_{\xi i-1 j}\right)\right]^{n}
$$

The second addend $R H S_{\eta i j}^{n}$ is written the same way.

The vector of mass concentrations of the mixture is determined using a scalar sweep. For approximation of the first derivatives in the system (3.7) the differences against the flow with the first order of accuracy are used and for the second derivatives, the central differences with the second order of accuracy are used. The temperature is determined in accordance with the [16].

\section{Results and analysis}

The calculations were carried out on a grid separated by spatial coordinates with the next parameters:

$$
2 \leq M_{\infty} \leq 6,2 \leq n \leq 15, \operatorname{Re}=10^{6}-10^{7}, \operatorname{Pr}=0,7
$$

To test the numerical method, the following experiment was performed: through the slit on the wall of $0.1 \mathrm{~cm}$ wide the hydrogen sound jet of $T_{0}=$ $800 K$ and $n=10.29$ was injected perpendicular to the main air flow (oxygen and nitrogen) of parameters $M_{\infty}=3.75, P_{\infty}=11090 \mathrm{~Pa}, T_{\infty}=$ $629.43 \mathrm{~K}, \operatorname{Pr}=0.7, \operatorname{Re}=62.73 \times 10^{6}$. The height and width were $H=15.2 \mathrm{sm}$ and $L=45 \mathrm{sm}$, respectively. Specific heat capacities at constant pressure $C_{p k}$ of $k$-component were calculated using fourth-order polynomial interpolation in temperature:

$$
C_{p k}=\sum_{i=1}^{5} a_{k i} T^{i-1}
$$

where $\left\{a_{k i}\right\}$ is an empirical constant defined for temperature within $300<T<5000 K$ [20].

Table 1 presents the sensitivity of the convergence of solutions to the characteristics of the grid by estimating the total $L_{1}^{\rho}=\frac{1}{N} \sum_{n=1}^{N} \varepsilon_{M}^{n}-\varepsilon_{\hat{M}}^{n} \mid$ and mean-square $L_{2}^{\rho}=\frac{1}{N} \sqrt{\sum_{n=1}^{N}\left|\varepsilon_{M}^{n}-\varepsilon_{\hat{M}}^{n}\right|^{2}}$ deviations of the norms of density residuals. Here $\varepsilon_{M}^{n}=\max _{(i, j) \in M}\left|\rho_{i, j}^{n+1}-\rho_{i, j}^{n}\right|, \quad \varepsilon_{\hat{M}}^{n}=\max _{(i, j) \in \hat{M}}\left|\rho_{i, j}^{n+1}-\rho_{i, j}^{n}\right| ; \quad \mathrm{N}$ is the number of iterations. Estimates were built for WENO and ENO schemes by successive refinement of the grids, and the grid $M$ with nodes $I \times J$ was taken as the initial one, then the nodes $M$ and $L$ of the grid $\hat{M}$ were varied. As follows from the table, the difference in the deviation of the values of the norms of the density residuals decreases with the grid refinement. The grid $\hat{M}$ with nodes $301 \times 281$ has minimal values while the indicator $L^{\rho}$ for the WENO scheme is less than that of the ENO scheme.

Table 1 - The relationship between the total and standard deviation (the density difference) on the size of the grid for WENO and ENO schemes

\begin{tabular}{|c|c|c|c|c|c|}
\hline \multicolumn{2}{|c|}{ The number of nodes } & WENO & ENO & WENO & ENO \\
\cline { 1 - 4 }$M=I \times J$ & $\hat{M}=M \times L$ & $L_{1}^{\rho}$ & $L_{1}^{\rho}$ & $L_{2}^{\rho}$ & $L_{2}^{\rho}$ \\
\hline \multirow{2}{*}{$I=301}$, & $M=I, L=221$ & $6,09 \cdot 10^{-2}$ & $8,02 \cdot 10^{-2}$ & $1,14 \cdot 10^{-3}$ & $1,4 \cdot 10^{-3}$ \\
$J=261$ & $M=I, L=281$ & $3,37 \cdot 10^{-2}$ & $3,49 \cdot 10^{-2}$ & $6,27 \cdot 10^{-4}$ & $7,22 \cdot 10^{-4}$ \\
\hline \multirow{2}{*}{$\begin{array}{l}I=321, \\
J=281\end{array}$} & $M=301, L=J$ & $2,74 \cdot 10^{-2}$ & $3,87 \cdot 10^{-2}$ & $5,29 \cdot 10^{-4}$ & $8,03 \cdot 10^{-4}$ \\
\cline { 2 - 5 } & $M=341, L=J$ & $2,91 \cdot 10^{-2}$ & $3,43 \cdot 10^{-2}$ & $6,11 \cdot 10^{-4}$ & $7,86 \cdot 10^{-4}$
\end{tabular}

International Journal of Mathematics and Physics 9, №2, 72 (2018) 
To compare with experimental data, the problem of a flat supersonic air flow $\left(M_{\infty}=2.9\right.$, $\left.P_{\infty}=0.0663 \mathrm{MPa}, T_{\infty}=108 \mathrm{~K}\right)$ with perpendicular injection of a helium sound jet $\left(T_{0}=217 \mathrm{~K}\right.$, $\left.P_{0}=1.24 \mathrm{MPa}\right)$ through a slit on the bottom wall of $0.0559 \mathrm{sm}$ width was calculated. The heights and width of the channel were $H=7,62 \mathrm{sm}$ and $L=25 \mathrm{sm}$, respectively. In this experiment it is necessary to know the $C_{p k}(\mathrm{~J} /(\mathrm{Mol} . \mathrm{K}))$ component of $\mathrm{O}_{2}, N_{2}, H_{e}$ at a temperature below $300 \mathrm{~K}$. Here, the experimental data of [21] are used (Table 2) in which the dependences of $C_{p k}$ on low temperatures are given. The construction of polynomial (4.1) is carried out using the method of least squares. For this purpose a system of linear algebraic equations is constructed for unknown empirical constants $\left\{a_{k i}\right\}$, which is solved by the LU decomposition method. The values $\left\{\alpha_{k i}\right\}$ found in this way are shown in Table 3 .

Table 2 - Thermo-chemical table (J / (Mol.K))

\begin{tabular}{|c|c|c|c|}
\hline $\mathrm{T},{ }^{0} \mathrm{~K}$ & $\mathrm{C}_{\mathrm{P} 1}\left(\mathrm{O}_{2}\right)$ & $\mathrm{C}_{\mathrm{P} 2}\left(\mathrm{~N}_{2}\right)$ & $\mathrm{C}_{\mathrm{P} 2}\left(\mathrm{H}_{\mathrm{e}}\right)$ \\
\hline 100 & ----- & 30.04253461 & 20.9336046 \\
\hline 120 & 29.66933952 & ---- & 20.9336046 \\
\hline 140 & ----- & 29.38966128 & 20.9336046 \\
\hline 150 & 29.40808320 & ---- & 20.9336046 \\
\hline 170 & 29.33305574 & ---- & 20.9336046 \\
\hline 180 & ----- & 29.25719093 & 20.9336046 \\
\hline 210 & 29.27142605 & ---- & 20.9336046 \\
\hline 220 & ----- & 29.20326494 & 20.9336046 \\
\hline 250 & 29.29822157 & ---- & 20.9336046 \\
\hline 260 & ---- & 29.17747260 & 20.9336046 \\
\hline 300 & 29.45497536 & 29.17161274 & \\
\hline
\end{tabular}

Table 3 - The values of of the empirical constants $a_{k i}$

\begin{tabular}{|c|c|c|c|}
\hline$T<300 K$ & $\mathrm{O}_{2}$ & $\mathrm{~N}_{2}$ & $\mathrm{H}_{\mathrm{e}}$ \\
\hline$a_{k 3}$ & $0.34843577 \mathrm{E}+02$ & $0.39755287 \mathrm{E}+02$ & $0.209336046 \mathrm{E}+02$ \\
\hline$a_{k 2}$ & $-0.90674514 \mathrm{E}-01$ & $-0.20027529 \mathrm{E}+00$ & $0.000000000 \mathrm{E}+00$ \\
\hline$a_{k 3}$ & $0.56072001 \mathrm{E}-03$ & $0.14293081 \mathrm{E}-02$ & $0.000000000 \mathrm{E}+00$ \\
\hline$a_{k 4}$ & $-0.15761941 \mathrm{E}-05$ & $-0.45017727 \mathrm{E}-05$ & $0.000000000 \mathrm{E}+00$ \\
\hline$a_{k \mathbf{B}}$ & $0.17168133 \mathrm{E}-08$ & $0.52356848 \mathrm{E}-08$ & $0.000000000 \mathrm{E}+00$ \\
\hline
\end{tabular}

Figure 2 shows the calculated and measured values of the pressure distribution on the wall in front of the slit and behind it. Curves 1, 2 are numerical calculations of WENO and ENO schemes, respectively; $\cdots$ is an experiment from [1]. The figure shows good agreement between the numerical and experimental data in front of the slit. However, behind the slit the values were underestimated. The curve obtained using the WENO scheme is located below the curve corresponding to the experiments and the curve obtained using the ENO scheme, because of the dissipative properties of the WENO scheme. In general, there is satisfactory agreement between the results of calculations and experimental measurements. 


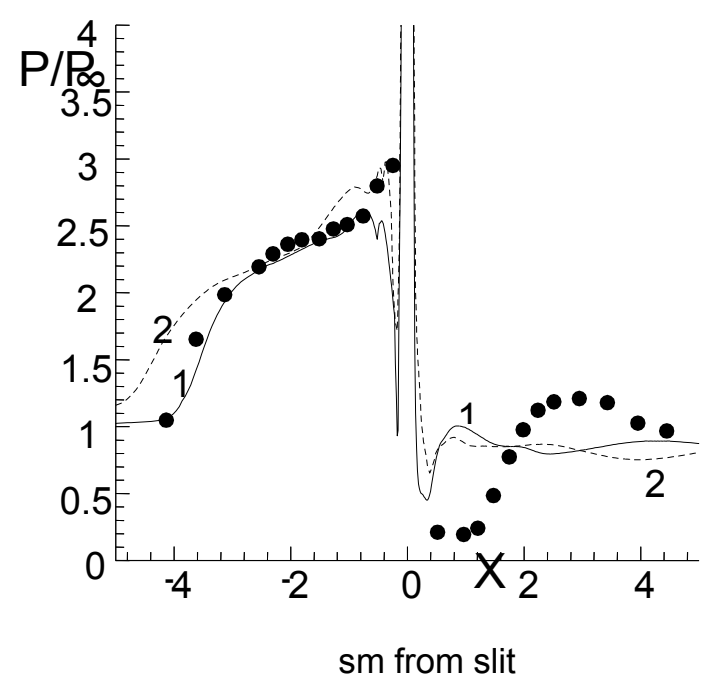

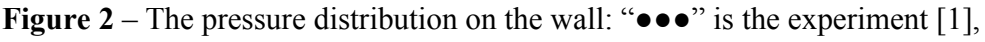
curve 1 is the WENO scheme, curve 2 is the ENO scheme

In Figures 3-6, the results of calculations of the problem of a flat supersonic air flow with transverse injection of a hydrogen sound jet through a slit on the bottom wall are given. The slit sizes of the injection jet are varied with three cases of the physical width of the slit $\left(h_{1}, h_{2}, h_{3}\right)$. In the first case, the dimensionless parameters of the computational domain are as follows: $h=1, L=$ $125, H=20$, and, for the second case: $h=1, L=$ $100, H=16$, which corresponds to $h_{2}=1.33 h_{1}$, and for the third case: $L=75, H=12, h_{3}=1.66 h_{1}$.

The parameters of oncoming flow are: $M_{\infty}=$ 3.75, $T_{\infty}=629.43 \mathrm{~K}, \operatorname{Pr}=0.7, \operatorname{Re}=10^{6}$; the parameters of the jet are: $T_{0}=800 \mathrm{~K}, n=15$ The width of the boundary layer on the input section on walls is $\delta=0.38 / h_{i}$, where $i=1,2,3$.

When an underexpanded jet flows into a stream, the pressure in the jet tends to equalize with the ambient pressure as a result of which the jet expands with the formation of a family of rarefaction waves that move to the jet boundaries forming a hanging shock which is closed by the Mach disk. The distribution of the local Mach number $\left(M=\sqrt{u^{2}+w^{2}} / c\right.$, with $c$ being a local velocity of sound, is shown in Figure 3 ( $3 a-h_{1}, 3 b-$ $\left.h_{2}, 3 \mathrm{c}-h_{3}\right)$. It follows from the graph that for $h_{1}$ (Figure 3a) the jet injected at a sonic speed accelerates and goes into a supersonic zone at some distance (the value of the local Mach number increases to 2.5), then the boundary of the resulting supersonic zone closes, delineating a circle. This circle corresponds to the barrel separating the supersonic zone from the subsonic one. At the top of the barrel the compression waves converge forming a Mach disk. Behind the barrel the flow slows down and becomes subsonic with a Mach number of 0.4. Ultimately, the flow of the jet accelerates and goes into the supersonic zone and the Mach number increases to 3 and above. With an increase in the slit width, a noticeable increase in the size of the circle is observed which can be traced from the comparison of Figures $3 \mathrm{a}$ and Figures 3b. However, in Figure 3c, the narrowing of the barrel is clearly observed despite the fact that here the slit size is maximum. The graphs clearly show that the size of the Mach disk varies in an ambiguous manner although it is known that an increase in the size of the slit leads to an expansion of the barrel-like structure in the jet and, consequently, to an increase in the Mach disk. Such an increase in the size of the Mach disk is explained in the case of $h_{3}$ a shift of the detachment zone towards the input boundary, the distance between the jet and the upper return area, respectively, becomes minimal (it is a confuser). As a result, the oncoming flow is significantly accelerated, thereby preventing the expansion of the jet. 
$\mathrm{a}$

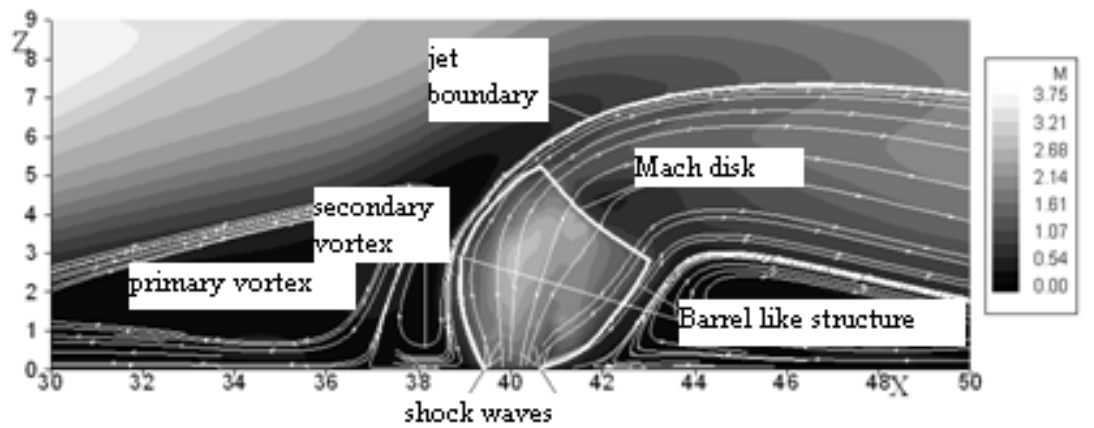

b
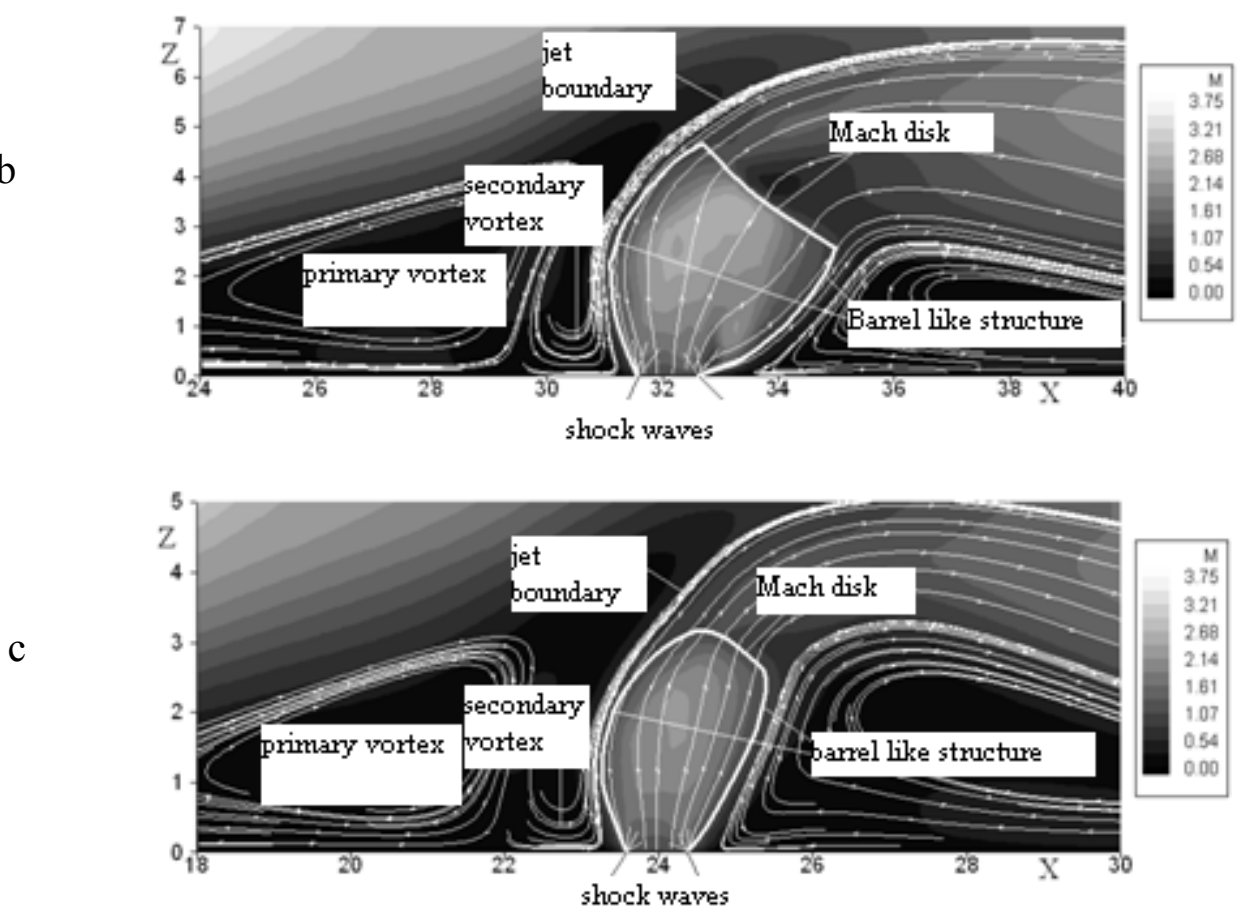

Figure 3 - The distribution of the local Mach number for the slit width:

$$
\text { a) } h_{1}, \text { b) } h_{2} \text {, c) } h_{3}
$$

The results of numerical experiments for the velocity vector field and streamlines, Figure 4 (4a$h_{1}, 4 \mathrm{~b}-h_{2}, 4 \mathrm{c}-h_{3}$ ), show a picture of the vortex structure arising from the interaction of shock waves with both the lower boundary layer and the upper one. The graph shows that in the zone adjoining to the wall, two vortices are formed in front of the jet which formed as a result of the primary and secondary separation of the flow from the wall. The directions of movement in them are opposite due to part of the air flow directly near the wall passing the sections of the oblique shock (2) and closing (3) turns down to the wall and penetrates into the zone of detached flow, then spreads in opposite directions. In this case, the vortex located closer to the jet moves counterclockwise, and the vortex located at a greater distance from it moves clockwise which is observed in the calculation results. Behind the jet the occurrence of a vortex region is associated with a zone of low pressures. The graphs show the region of separation on the upper wall due to the interaction of the head shock wave (1) with the upper boundary layer. The qualitative pictures of the return zones are similar in all three cases. However, they are quantitatively different since dimensions increase significantly with increasing of the slit width.

In Figure 4a-c in addition to the known ones, there is an additional vortex on the bottom wall at some distance behind the jet which was 
formed as a result of the interaction of the shock (6) with the bottom boundary layer. As the size of the slit increases, it shifts towards the jet (comparison of Figure 4a-b). Thus, we can conclude that an increase in the size of the slit will lead to an increase in the mixing of air and fuel directly behind the injected jet due to the extensive zones of return flows.
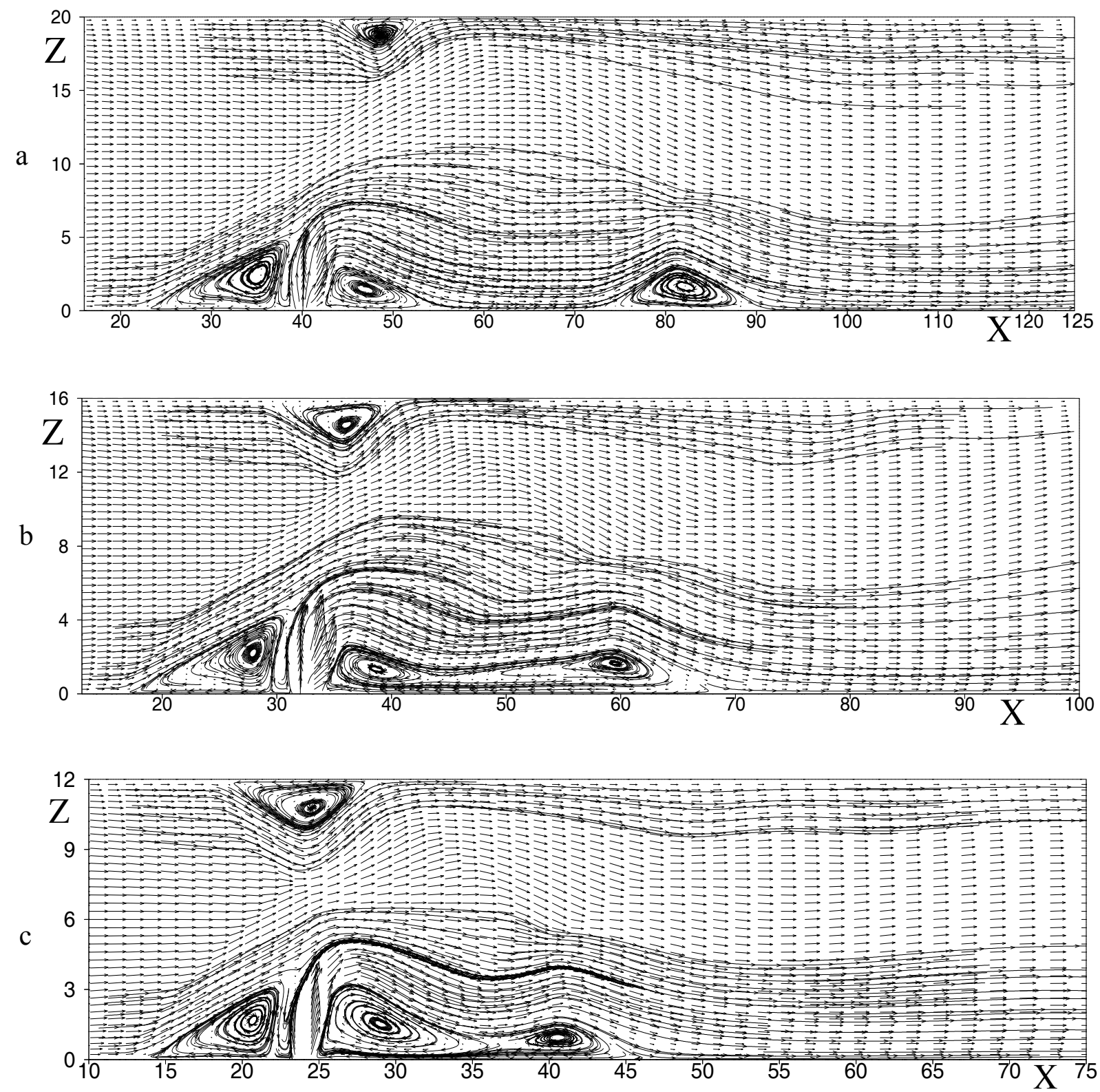

Figure 4 - The velocity vectors and streamlines for the slit width:

$$
\text { a) } h_{1}, \text { b) } h_{2}, \text { c) } h_{s}
$$

Figure 5 a-c shows the distribution of the mass concentration of hydrogen for all three considered slits. The contours of a constant concentration of hydrogen show that the maximum concentration value is reached behind the jet. The results show that increasing the size of the slit affects the range of the injected jet, so with $h_{1}$ the line of $1 \%$ hydrogen concentration rose to a height of $45 \%$ of the total height of the channel (Figure 5a), when with $h_{2}$ the same line corresponds to half of the height of the channel, but for $h_{3}$ is $47.5 \%$ of the height of the channel. In this case, a decrease in the range of the 
injected hydrogen also indicates a blockage of the jet.

The pressure distribution on the bottom wall (Figure 6a) shows that the excess pressure in front of the jet increases and at some distance behind the jet its growth is also observed due to the detachment of the boundary layer. At the same time, it is noticeable from the graph that the curves for large $h$ are significantly higher. A similar pattern is observed on the upper wall (Figure 6b).

a

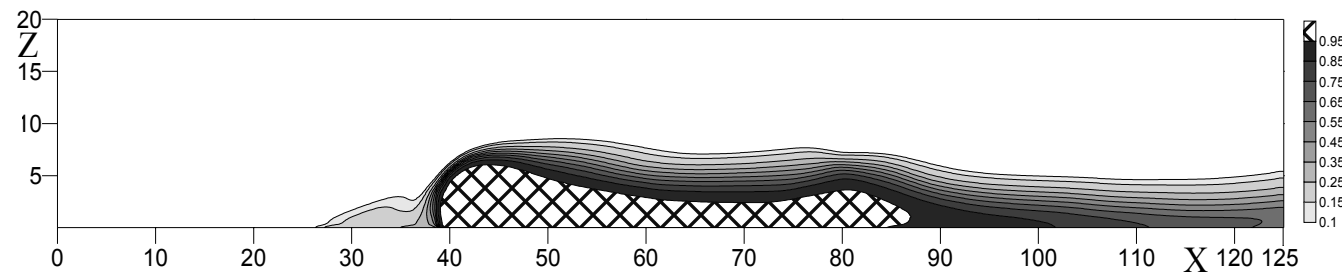

$\mathrm{b}$
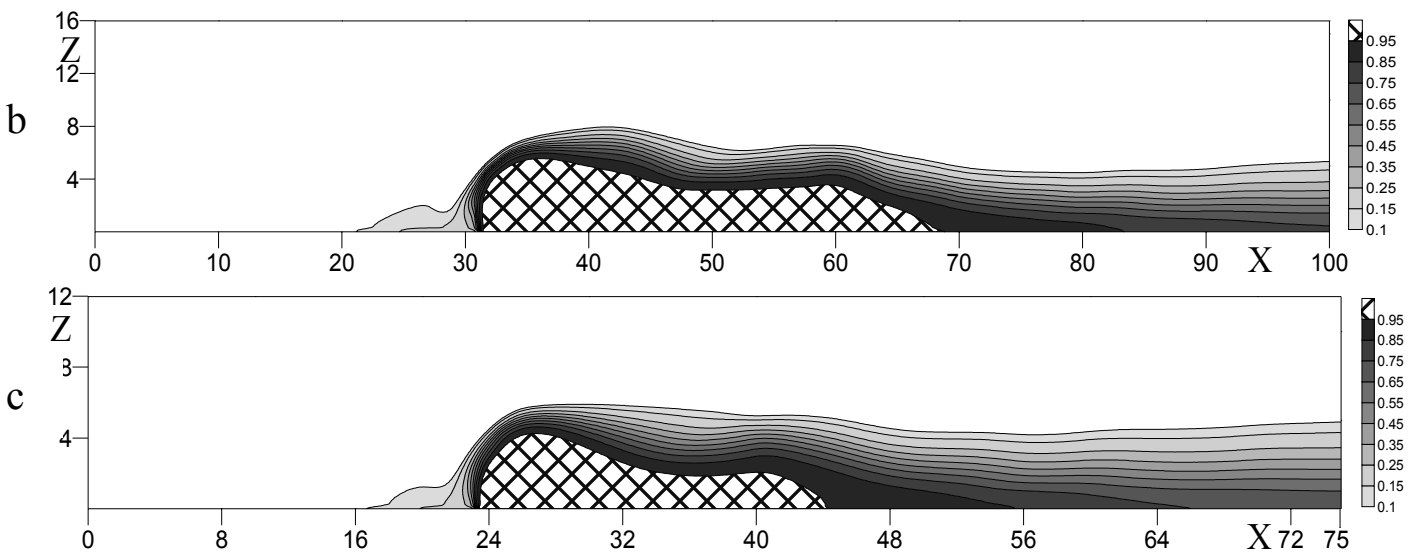

Figure 5 - The distribution of the mass concentration of hydrogen for the slit width:

a) $h_{1}$, b) $h_{2}$, c) $h_{3}$
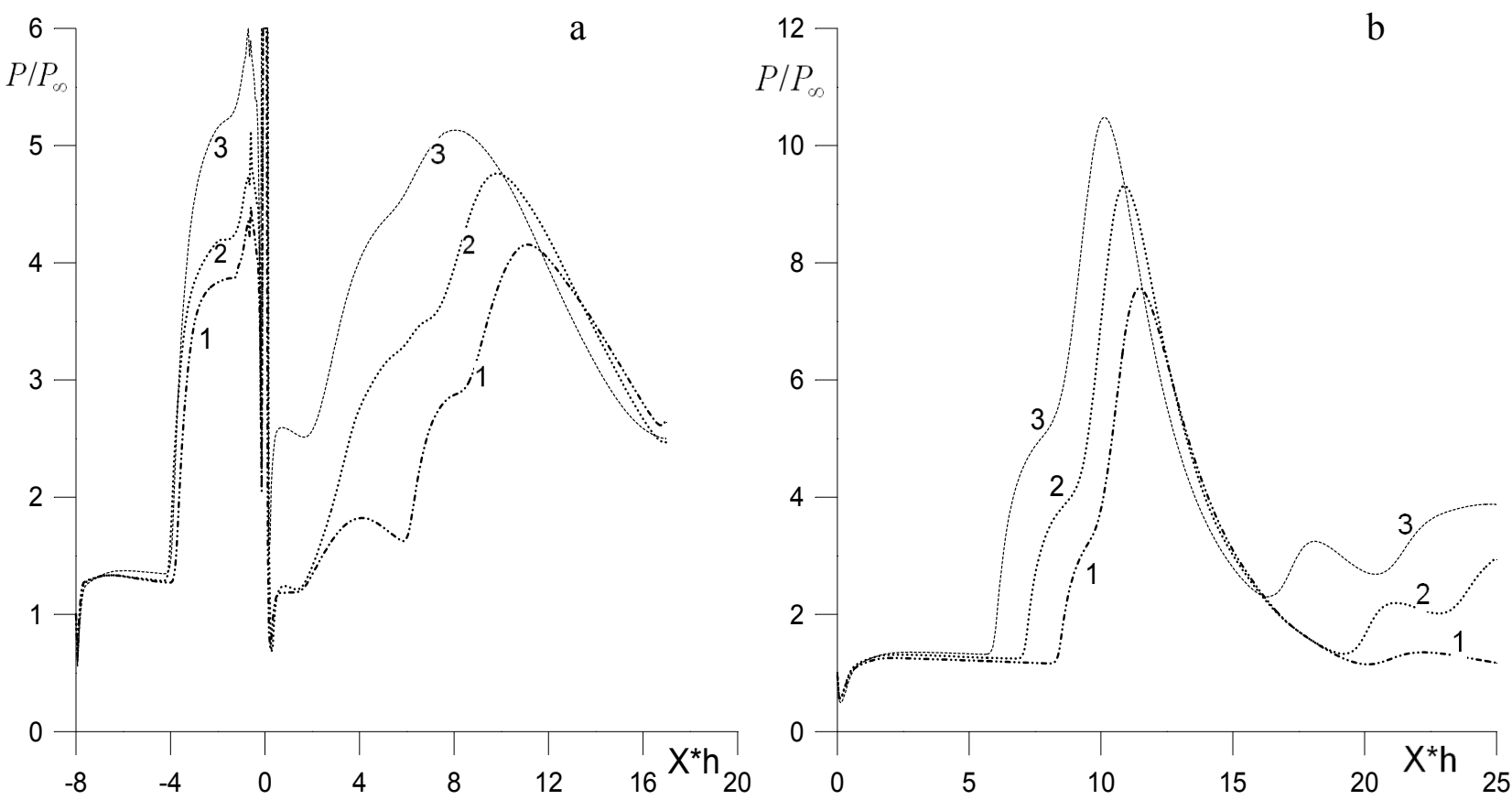

Figure 6 - The pressure distribution on the bottom and upper walls

International Journal of Mathematics and Physics 9, №2, 72 (2018) 


\section{Conclusion}

Using a numerical experiment, a shock-wave structure and a detachment region are obtained due to the interaction of shock waves with the boundary layer on both the upper and lower walls of the channel and depending on the size of the injected jet. An ambiguous decrease is shown in the expansion of the jet with an increase in the width of the slit which is a consequence of a confuser between the injected jet and the detachment zone on the upper wall. In addition to the well-known shockwave pattern an additional structure appeared here due to the interaction of the reflected shock (6) with the boundary layer on the bottom wall at some distance behind the jet which can lead to an increase in air and fuel mixing due to the extensive return flow zones. It is established that, depending on the increase of $h$, the range of the jet decreases due to its locking. Excessive pressure on the walls increases with increasing jet size which leads to an increase in the load on the channel walls. Comparison of calculations with experimental data shows satisfactory agreement of results. Thus, the numerical model developed in this paper for calculating turbulent flows based on the WENO scheme allows one to study both the supersonic flow of a multicomponent gas and to simulate combustion processes.

\section{References}

1. Shun J., Sh.., Yun S. "Chislenoe issledovanie techenii s khimicheskimi reaktsiyami na osnove LUfaktorizovannoi skhemi, postroennoi metodom simetrichnoi posledovatelnoi verkhnei relaksatsii [Numerical study of flows with chemical reactions based on the LU-factorized scheme, constructed by the method of symmetric sequential upper relaxation]." Aerospace Engineering 10 (1990): 102-113.

2. Chenault F.C., Beran P.S. " $k \quad \varepsilon$ and Reynolds Stress Turbulence Model Comparsions for Two-Dimensional Injection Flows." AIAA Journal 36, no. 8 (1998): 1401-1412.

3.Aso S., Inoue K., Yamaguchi K., Tani Y. "A study on supersonic mixing de circular nozzle with various injection angles for air breathing engine." Acta Astronautica 65 (2009): 687-695.

4. Spade F., Zukoski Y. "Issledovanie vzaimodeistviya gazovoi strui, vitekayushei iz poperechnoi sheli, so sverkhzvukovim potokom [Investigation of the interaction of a gas jet flowing from a transverse slit with a supersonic flow]." Rocket technology and astronautics 2 (1968): 16-25.

5. Avduyevskii V.S., Medvedev K.I., Polyanskii M.N. "Vzaimodeistvie sverkhzvukovogo potoka s poperechnoi stuei, vduvaemoi cherez krugloe otverstie $\mathrm{v}$ plastine [The interaction of supersonic flow with a transverse jet blown through a round hole in the plate]." News of the USSR Academy of Sciences. Fluid and gas mechanics 5 (1970): 193197.

6. Glagolev A.I., Zubkov A.I., Panov U.A. "Vzaimodeistvie strui gaza, vytekayushei iz otverstiya $\mathrm{v}$ plastine, so sverkhzvukovim potokom [The interaction of the gas jet flowing out of the hole in the plate with supersonic flow]." News of the USSR Academy of Sciences. Fluid and gas mechanics 2 (1968): 99-102.

7. Shets D., Hoskins P.F., Leman H. "Structura poperechnikh strui, istekayushikh v sverkhzvukovoi potok $\mathrm{s}$ bolshoi stepenyu nedorasshireniya [The structure of transverse jets flowing into a supersonic flow with a high degree of under-expansion]." Rocket technology and astronautics 5 (1967): 65-69.

8. Huang W., Zhen-guo Wang, Wu J., Shi-bin $\mathrm{Li}$ "Numerical prediction on the interaction between the incident shock wave and the transverse slot injection in supersonic flows." Aerospace Science and Technology 1.86 (2012): 1-9.

9. Kim J.H., Yoon Y., Jeung I.S., Huh H., Choi J.Y. "Numerical study of mixing enhancement by shock waves in model scramjet engine." AIAA Journal 41 (6) (2003): 1074-1080.

10.Liou W.W., Huang G., Shih T.H. "Turbulence model assessment for shock wave/turbulent boundary-layer interaction in transonic and supersonic flows." Computers \& Fluids no. 29 (2000): 275-299.

11.Knight D., Yan H., Panaras A., Zheltovodov A. "RTO WG 10: CFD validation for shock wave turbulent boundary layer interactions." AIAA Paper N 2002-0437.

12.Fyodorova N.N., Fedorchenko I.A. "Raschyot vzaimodeistviya padayushego kosogo skachka uplotneniya s turbulentnim pogranichnim sloem na plastine [Calculation of the interaction of a falling oblique shock wave with a turbulent boundary layer on a plate]." Applied mechanics and technical physics 45, no. 3 (2004): 61-71.

13. Harten A., Osher S., Engquist B., Chakravarthy S.R. "Some Results on Uniformly High-Order Accurate Essentially Non-Oscillatory Schemes." Applied Num. Math. no. 2 (1986): 347377. 
14. Yershov S.V. "Kvazimonotonnaya ENO skhema povishennoi tochnosti dlya integrirovaniya uravnenii Eulera i Navie-Stoksa [Quasi-monotone ENO-scheme of increased accuracy for integrating the Euler and Navier-Stokes equations]." Math modeling 6, no. 11 (1994): 63-75.

15. Yang J.Y. "Third Order Non-Oscillatory Schemes for the Euler Equations." AIAA Journal 29, no. 10 (1991): 1611-1618.

16. Beketayeva A.O., Naimanova A.Z. "Primenenie ENO (Essentially Non-Oscillatory) skhemi dlya modelirovaniya techeniya mnogokomponentnoi gazovoi smesi [The use the ENO (Essentially Non-Oscillatory) Scheme to Simulate a Multicomponent Gas Mixture]." Computational Technologies 12, no. 4 (2007): 17-25.

17. Poinsot T.J., Lele S.K. "Boundary Conditions for Direct Simulation of Compressible
Viscous Flows." Journ. of Comput. Phys. no. 101 (1992): 104-129.

18. Bruel P., Naimanova A.Zh. "Computation of the normal injection of a hydrogen jet into a supersonic air flow." Thermophysics and Aeromechanics 17, no. 4 (2010): 531-541.

19. Liu X., Osher S., and Chan T. "Weighted Essentially Non- oscillatory Schemes." Journal of Computational Physics 115 (1994): 200-212.

20. Kee R.J., Rupley F.M., Miller J.A. "CHEMKIN-II: a FORTRAN chemical kinetic package for the analysis of gas-phase chemical kinetics." SANDIA Report SAND89-8009 (1989).

21. Kikoina I. K. "Tablitsi fizicheskih velichin [Physical Parameters Tables]." Moscow: Atomizdat (1976): 1008. 